\title{
Anti-Tuberculosis Drug Induced Hepatotoxicity and Associated Factors among Tuberculosis Patients at Selected Hospitals, Ethiopia
}

This article was published in the following Dove Press journal:

Hepatic Medicine: Evidence and Research

\section{Yalew Molla (iD \\ Muluken Wubetu \\ Bekalu Dessie (D)}

Pharmacy Department, Health Science College, Debre Markos University, Debre Markos, Ethiopia
Correspondence: Yalew Molla Pharmacy Department, Debre Markos University, PO Box, 269, Debre Markos, Ethiopia

Email yalew.molla2009@gmail.com
Background: Tuberculosis caused by susceptible mycobacterium tuberculosis strains is effectively treated by the first-line anti-tuberculosis drugs. However, most antibacterial drugs are known to induce hepatotoxicity which may limit their adherence and hence lead to the development of mycobacterial drug resistance.

Objective: The aim of this study was to assess the incidence of anti-tuberculosis drug induced hepatotoxicity and associated factors among tuberculosis patients of Debre Markos, Mota, and Bichena Hospitals.

Methods: The prospective cross sectional-study was conducted in three hospitals of East Gojjam zone by taking blood samples of new tuberculosis patients every 2 weeks for 2 months to measure the elevation of liver proteins indicating liver toxicity from the onset of starting therapy. A semi-structured questionnaire was also used to collect the socio-demographic data and factors of anti-tubeculosis drug induced liver toxicity. To identify factors associated with drug induced hepatotoxicity, binary logistic regression followed by multivariate analysis was applied at a statistically significant level of $P<0.05$.

Results: The incidence of hepatotoxicity among tuberculosis patients is $7.9 \%$. Diagnosis of extrapulmonary tuberculosis, having comorbid disease, and old age are significantly associated $(P<0.05)$ with first-line antituberculosis drugs induced hepatotoxicity.

Conclusion: The incidence of hepatotoxicity is relatively high among tuberculosis patients taking first-line anti-tuberculosis drugs. Therefore, the liver function of patients with old age, comorbid diseases, and extrapulmonary tuberculosis should be regularly monitored to reduce the severity of drug-induced hepatotoxicity.

Keywords: anti-tuberculosis drugs, hepatotoxicity, tuberculosis

\section{Introduction}

Tuberculosis (TB) is a chronic bacterial infection caused by Mycobacterium tuberculosis complex, most commonly by Mycobacterium tuberculosis, and is usually characterized pathologically by the formation of granulomas. The most common

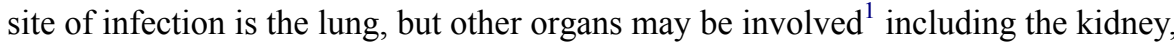
spine, and brain, skin, etc. ${ }^{2,3}$

The cornerstone of TB management is a 6-month course of using anti-TB drugs where isoniazid, rifampicin, pyrazinamide, and ethambutol are taken for 2 months in the intensive phase followed by a fourth month use of isoniazid and rifampicin in the continuous phase of managing protocols of the disease. Compliance is crucial 
for curing TB. Adverse effects often negatively affect the compliance, because they frequently require a change of treatment, which may have negative consequences for treatment outcome. One of the adverse effects affecting TB treatment outcome is anti-TB drug induced hepatotoxicity. ${ }^{4}$

Hepatotoxicity is usually presented and diagnosed with jaundice or a high concentration of liver function marker proteins like aspartate aminotransferase (AST)/ alanine aminotransferase (ALT), alkaline phosphatase (APT), or total bilirubin. Treatment should be interrupted and, generally, a modified or alternative regimen should be used for those with ALT elevation more than three times the upper limit of normal (ULN) in the presence of hepatitis symptoms and/or jaundice, or five times the ULN in the absence of symptoms. An increase in serum ALT is more specific for hepatocellular injury than an increase in AST which can also signify abnormalities in muscle, heart, or kidney. ${ }^{5}$

Among the first-line anti-TB drugs, isoniazid, rifampicin, and pyrazinamide are known to cause hepatotoxicity, but pyrazinamide attribute to a higher percentage for the drug induced liver toxicity compared to the other drugs. ${ }^{6,7}$ The treatment regimen of tuberculosis can be tailored on patient's needs, mycobacterial tuberculosis resistance pattern, and location of the disease. ${ }^{8}$ Even though the first-line anti-TB drugs are effective, their liver toxicity may lead to drug interruption; which can in turn be the cause for the development of Multidrug Resistant Tuberculosis (MDR-TB). The simultaneous use of a number of drugs for a prolonged period of time, for the treatment of $\mathrm{TB}$, further complicates the drug-induced toxicity problem. ${ }^{9}$ This adverse effect of anti-TB drugs is associated with the mortality of $6-12 \%$ patients with TB. Therefore, the purpose of this study was to assess the magnitude of hepatotoxicity induced by anti-TB drugs and its associated factors among newly diagnosed TB patients at selected health facilities of Ethiopia.

\section{Methods}

\section{Study Design, Period, and Settings}

A cross-sectional prospective study was conducted from December 2017 to June 2019 in hospitals of Debre Markos, Mota and Bichena Hospitals.

\section{Study Population}

All patients who were newly-diagnosed for TB and became positive for the first time and started anti-TB medication from December 2017 to June 2019 in the study areas will be included in the study.

\section{Exclusion Criteria}

Patients taking antiretroviral therapy (ART) were excluded from the study. In addition, patients who had baseline ALT and AST values greater than two times the ULN (ie, $\mathrm{ULN}>40 \mathrm{U} / \mathrm{L}$ and $37 \mathrm{U} / \mathrm{L}$, respectively) were excluded. Moreover, those patients who become positive for Hepatitis C and/or B virus at the baseline test were also excluded from the study.

\section{Sample Size and Sampling Techniques}

The sample size was determined based on the previous prevalence result studied in Addis Ababa, Ethiopia ${ }^{10}$ at a significant level of 0.05 and 216 patents were taken proportionally from each hospital. Then, eligible candidates were selected by convenient sampling technique.

\section{Data Collection Procedure}

Socio-demographic data was collected from each participant using a semi-structured interviewer administered questionnaire. After completion of the interview, all respondents who gave verbal consent for biochemical tests were appointed in the early morning before starting the treatment regimen. Then, $3 \mathrm{~mL}$ of blood sample was collected after cleaning the cubital area by $70 \%$ ethanol by laboratory technologists. Blood samples from each patient were collected every 2 weeks after starting treatment in the intensive phase or in the first 2 months of managing protocols of TB. Therefore, each patient gave five samples of blood in the first 2 months of monitoring the TB, including the baseline blood sample. After collection, each blood sample was transferred into a serum separator tube and subjected to centrifuge to separate serum. Then, biochemical analysis of AST, ALT, APT, direct bilirubin, and total bilirubin was done using an automated spectrophotometric analyzer.

The anti-TB drugs regimen of each case was interrupted when the laboratory level of ALT and AST was found to be more than three times the upper limit of normal (ULN) in the 
presence of hepatitis symptoms and/or jaundice, or five times the ULN in the absence or presence of symptoms. The normal maximum values of ALT and AST were $40 \mathrm{U} / \mathrm{L}$ and $37 \mathrm{U} / \mathrm{L}$, respectively, which were the same cut-off point for both men and women.

After 2 weeks of the rehabilitation period, their liver function was tested and ALT and/or AST level has been found to be less than three times ULN without the manifestations of hepatotoxicity. These had led the authors to believe that the hepatotoxicity developed by the cases was considered to be caused by the first-line anti-TB drugs.

The developed hepatotoxicity was categorized into different grades based on the WHO grading system using ALT and/or AST levels. ${ }^{4}$

The treatment plan was decided by the respective physicians of each patient developing hepatotoxicity based on the laboratory findings of the blood biochemical liver function test and the manifestations complained by individual patient. Then, the treatment option of TB made by the physician for each patient developing hepatotoxicity was collected from the TB patients' registration book.

\section{Statistical Analysis}

Data was entered in Epi Info and analyzed with SPSS, version 20. The descriptive frequency of variables was determined using SPSS. A binary logistic regression model was used to see the association between each independent variable and the dependent variable. All variables with a $P$-value $\leq 0.25$ in the bivariable logistic regression analysis were entered into a multivariable logistic regression model for controlling confounders, further analysis, and to identify variables having a significant association with the dependent variable. An adjusted odds ratio with $95 \%$ confidence interval was used to determine the strength of association between dependent and independent variables both in the case of bivariable and multivariable logistic regression analyses. Variables having a $P$-value $<0.05$ in the multivariable logistic regression model were considered as significantly associated variables.

\section{Operational Definitions}

- Smoking cigarette: History of smoking practice by the patients before and after starting the ant-TB medications.

- Social drug use: The practice of using khat, marijuana, coffee, and tea.

- Alcohol use: Any practice of consuming alcohol by TB patients.
- Concomitant drug use: The use of other drugs for the treatment of concomitant disease at the time of using the anti-TB drugs.

\section{Results}

\section{Socio-Demographic and Behavioral Factors}

The majority of the TB patients taking anti-TB first-line drugs, 153 ( $70.8 \%$ belonged to the age range of $18-49$ years. More than two-thirds of the study participants $(69 \%)$ were married and $13 \%$ were single in marital status. Female study participants $(118,54.6 \%)$ were greater as compared to the male ones. Among the TB patients participated in the study, around two thirds of the study populations used alcohol (59.3\%) and coffee (69.4\%) (Table 1).

Table I Socio-Demographic and Behavioral Characteristics of TB Patients $(n=216)$ Taking First-Line Anti-TB Drugs at Three Public Hospitals of East Gojjam Zone from 2017-2019

\begin{tabular}{|c|c|c|c|}
\hline Variables & Categories & Frequency & Percentage \\
\hline \multirow[t]{3}{*}{ Age in years } & $18-49$ & 153 & 70.8 \\
\hline & $50-64$ & 54 & 25.0 \\
\hline & $\geq 65$ & 9 & 4.2 \\
\hline \multirow[t]{2}{*}{ Sex } & Male & 98 & 45.4 \\
\hline & Female & 118 & 54.6 \\
\hline \multirow[t]{2}{*}{ Residence } & Urban & 97 & 44.9 \\
\hline & Rural & 119 & 55.1 \\
\hline \multirow[t]{4}{*}{ Marital Status } & Single & 28 & 13.0 \\
\hline & Married & 149 & 69.0 \\
\hline & Divorced & 27 & 12.5 \\
\hline & Widowed & 12 & 5.6 \\
\hline \multirow{7}{*}{$\begin{array}{l}\text { Educational } \\
\text { Status }\end{array}$} & Unable to read and & 41 & 19.0 \\
\hline & write & & \\
\hline & Read and write & 37 & 17.1 \\
\hline & Primary School & 22 & 10.2 \\
\hline & Secondary School & 95 & 44.0 \\
\hline & College diploma & 14 & 6.5 \\
\hline & $\begin{array}{l}\text { University degree } \\
\text { and above }\end{array}$ & 7 & 3.2 \\
\hline Smoking & Yes & 10 & 4.6 \\
\hline Cigarette & No & 206 & 95.4 \\
\hline \multirow[t]{3}{*}{ Social drug use } & No & 45 & 20.8 \\
\hline & Coffee & 150 & 69.4 \\
\hline & Tea & 21 & 9.7 \\
\hline \multirow[t]{2}{*}{ Alcohol use } & Yes & 128 & 59.3 \\
\hline & No & 88 & 40.7 \\
\hline
\end{tabular}




\section{Clinical Features of Participants Developing Hepatotoxicity}

Among TB patients starting first-line anti-TB drugs, 17 (7.9\%) developed hepatotoxicity. Patients with extrapulmonary TB $(n=14)$, comorbidity with HIV/AIDS $(n=11)$, and low BMI $(n=13)$ had developed anti-TB drug induced hepatotoxicity (Table 2).

\section{Antituberculosis Drug Induced Hepatotoxicity Characteristics of the}

\section{Cases}

The majority of the cases developing hepatotoxicity were females. Twelve of the 17 cases had developed hepatotoxicity in the first months of intensive phases of treatment, whereas others had developed hepatotoxicity in the second months of the initiation phases of the full regimen. All of the cases manifested at least one of the major symptoms of hepatotoxicity. The level of hepatotoxicity was measured using the plasma level of AST and/or ALT with the sign and symptoms observed or complained by the individual participants developing hepatotoxicity. Based on the WHO hepatotoxicity grading system, ${ }^{4}$ more than half of the cases had developed grade three level of hepatotoxicity (Table 3).
Table 2 Clinical Factors of TB Patients $(n=216)$ Taking First-Line Anti-TB Drugs at Three Public Hospitals of East Gojjam Zone, 2017-2019

\begin{tabular}{|c|c|c|c|c|}
\hline \multirow[t]{2}{*}{ Variables } & \multirow[t]{2}{*}{ Category } & \multicolumn{2}{|c|}{ Hepatotoxicity } & \multirow[t]{2}{*}{ Total } \\
\hline & & Yes & No & \\
\hline Extent of TB & $\begin{array}{l}\text { Pulmonary TB } \\
\text { Extrapulmonary } \\
\text { TB }\end{array}$ & $\begin{array}{l}3 \\
14\end{array}$ & $\begin{array}{l}149 \\
50\end{array}$ & $\begin{array}{l}156 \\
60\end{array}$ \\
\hline Comorbidity & $\begin{array}{l}\text { HIV/AIDS } \\
\text { Hypertension } \\
\text { Diabetic mellitus } \\
\text { Kidney disease } \\
\text { No }\end{array}$ & $\begin{array}{l}11 \\
0 \\
0 \\
0 \\
6\end{array}$ & $\begin{array}{l}26 \\
6 \\
13 \\
8 \\
146\end{array}$ & $\begin{array}{l}37 \\
6 \\
13 \\
8 \\
152\end{array}$ \\
\hline BMI & $\begin{array}{l}<18.5 \\
18.5-24.9 \\
25-29.9 \\
\geq 30\end{array}$ & $\begin{array}{l}13 \\
4 \\
0 \\
0\end{array}$ & $\begin{array}{l}44 \\
133 \\
21 \\
1\end{array}$ & $\begin{array}{l}56 \\
138 \\
21 \\
1\end{array}$ \\
\hline $\begin{array}{l}\text { Concomitant drug } \\
\text { use }\end{array}$ & $\begin{array}{l}\text { Yes } \\
\text { No }\end{array}$ & $\begin{array}{l}2 \\
15\end{array}$ & $\begin{array}{l}24 \\
175\end{array}$ & $\begin{array}{l}26 \\
190\end{array}$ \\
\hline
\end{tabular}

\section{Symptoms and Managements of Hepatotoxicity Provided}

As presented in Table 4, all of the TB patients developing hepatotoxicity had complained of nausea and/or vomiting.

Table 3 Description of Drug Induced Hepatotoxicity Among Cases $(n=17)$ Taking First-Line Anti-TB Drugs at Three Public Hospitals of East Gojjam Zone, 2017-2019

\begin{tabular}{|l|l|l|l|l|l|l|l|}
\hline Cases & Sex & Age & $\begin{array}{l}\text { Time } \\
\text { (Weeks) }\end{array}$ & Symptoms & $\begin{array}{l}\text { Peak ALT and/or } \\
\text { AST (U/L) }\end{array}$ & $\begin{array}{l}\text { Peak Total } \\
\text { Bilirubin (mg/dL) }\end{array}$ & $\begin{array}{l}\text { Grades of Hepatotoxicity/Based } \\
\text { on ALT/AST Level/ }\end{array}$ \\
\hline I & Female & 42 & 2nd & Yes & 300 & 1.9 & 3 \\
2 & Female & 45 & 2nd & Yes & 297 & 2 & 3 \\
3 & Male & 50 & 2nd & Yes & 245 & 1.1 & 3 \\
4 & Female & 55 & 6th & Yes & 402 & 1.9 & 4 \\
5 & Male & 57 & 8th & Yes & 489 & 2 & 4 \\
6 & Male & 65 & 4 th & Yes & 455 & 2 & 4 \\
7 & Female & 79 & 4 th & Yes & 475 & 1.8 & 4 \\
8 & Female & 60 & 8th & Yes & 456 & 2 & 4 \\
9 & Female & 67 & 2nd & Yes & 432 & 1.9 & 4 \\
10 & Male & 42 & 6th & Yes & 412 & 1.7 & 3 \\
II & Female & 65 & 2nd & Yes & 319 & 3 & 2 \\
I2 & Female & 55 & 2nd & Yes & 130 & 1.2 & 3 \\
13 & Female & 18 & 6th & Yes & 320 & 1.1 & 3 \\
I4 & Male & 27 & 4th & No & 256 & 1.5 & 3 \\
I5 & Male & 31 & 2nd & Yes & 355 & 1.3 & 2 \\
16 & Female & 32 & 2nd & Yes & 185 & 1.8 & 3 \\
17 & Male & 44 & 2nd & No & 284 & & 4 \\
\hline
\end{tabular}


Table 4 Symptoms and Managements Given to the Cases $(n=17)$ Taking First-Line Anti-TB Drugs at Three Public Hospitals of East Gojjam Zone, 2017-2019

\begin{tabular}{|l|l|l|}
\hline Items & & $\mathbf{N}(\%)$ \\
\hline Symptoms & Nausea and vomiting & $17(100)$ \\
& Abdominal pain & $2(11.76)$ \\
& Malaise & $8(47.10)$ \\
& Jaundice & $5(29.41)$ \\
\hline Management & $\begin{array}{l}\text { Discontinued and rechallenged with the } \\
\text { previous full regimen } \\
\end{array}$ & $17(100)$ \\
& $\begin{array}{l}\text { Discontinued and rechallenged with the } \\
\text { safest individual drug regimen }\end{array}$ & $0(0)$ \\
\hline
\end{tabular}

In addition, nearly half $(8(47.10 \%))$ of the hepatotoxicity developed cases had complained of malaise.

Based on the severity of hepatotoxicity developed, 17 cases were managed similarly where the regimen was discontinued for 2 weeks of rehabilitation purpose. Then, the initial regimen of each cases was re-initiated without changing the types or doses of anti-TB drugs after checking the level of the AST/ALT of each case with strict follow-up (Table 4).

\section{Univariate and Multivariate Analysis of Risk Factors Associated with the Development of Hepatotoxicity}

Extrapulmonary TB, having a comorbid condition, alcohol consumption, and being geriatrics ( $\geq 65$ years old) were found to be associated with increased risk of developing anti-TB drugs induced hepatotoxicity on bivariate logistic regression analyses. However, on multivariable logistic regression analyses, extra TB, old age, and having comorbid condition were found to be significant predictors $(P<0.05)$ in the development of anti-TB drugs induced hepatotoxicity (Table 5).

The likelihood of developing hepatotoxicity to anti-TB drugs was 0.85 times $(\mathrm{AOR}=0.077 ; 95 \% \mathrm{CI}=0.008-0.74$ ) lower in patients aged 18-49 years old compared to those aged 65 or above.

Patients with pulmonary $\mathrm{TB}$ had 0.26 ( $\mathrm{AOR}=0.74$; 95\% $\mathrm{CI}=0.015-0.36$ ) times lower risk of developing hepatotoxicity to anti-TB drugs compared to those with extrapulmonary TB. Patients who had a comorbid medical condition were 12.9-times (AOR $=12.9 ; 95 \% \mathrm{CI}=2.45$ 67.60) more likely to experience hepatotoxicity compared to nurses who had no comorbidity (Table 5).

\section{Discussion}

The incidence of first-line anti-TB induced hepatotoxicity in the present study was found to be $8.9 \%$, comparable to a study conducted in Malaysia (9.7\%). ${ }^{6}$ In addition, the incidence of hepatotoxicity in the present study was comparable to those reported by a study conducted in Dawro zone, Ethiopia (8.1\%). ${ }^{11}$ However, the finding of this study was slightly higher than the report in Iran (5.5\%). ${ }^{12}$ In addition, the anti-TB induced hepatotoxicity of the present study was lower than studies conducted in Pakistan (13\%), Spain (12\%) Florida (16.4\%), and Morocco (24.6\%). ${ }^{9,-13-15}$ The reason for the discrepancy in the incidence of hepatotoxicity among newly diagnosed TB patients in different literatures might be related to the differences in the lifestyle, nutrition status, comorbid condition, genetic make-up, and use of different drugs, in addition to the anti-TB first-line drugs. ${ }^{12,15,16}$

The anti-TB drugs induced hepatotoxicity of the cases happened in times ranging from 8-56 days of starting the

Table 5 Univariate and Multivariate Analysis of Risk Factors Associated with the Development of Hepatotoxicity Among TB Patients at Three Public Hospitals of East Gojjam Zone, 2017-2019

\begin{tabular}{|c|c|c|c|c|c|c|c|c|}
\hline \multirow[t]{2}{*}{ Variables } & \multirow[t]{2}{*}{ Category } & \multicolumn{2}{|c|}{ Hepatotoxicity } & \multicolumn{2}{|c|}{ Bivariate Analysis } & \multicolumn{3}{|c|}{ Multivariate Analysis } \\
\hline & & Yes & No & COR & $P$-value & AOR & $P$-value & $95 \% \mathrm{Cl}$ \\
\hline \multirow[t]{3}{*}{ Age } & $18-49$ & 8 & 145 & 14.5 & 0.000 & 0.077 & 0.026 & $(0.008-0.74)$ \\
\hline & $50-64$ & 5 & 49 & 7.84 & 0.012 & 0.17 & 0.151 & $(0.015-1.91)$ \\
\hline & $\geq 65$ & 4 & 5 & 1 & I & 1 & I & \\
\hline \multirow[t]{2}{*}{ Diagnosis of TB } & Pulmonary & 3 & 149 & 13.9 & 0.000 & 0.74 & 0.001 & $(0.015-0.36)$ \\
\hline & Extrapulmonary & 14 & 50 & 1 & 1 & 1 & 1 & \\
\hline \multirow[t]{2}{*}{ Alcohol } & Yes & 15 & 113 & 0.18 & 0.023 & 2.8 & 0.23 & $(0.53-15.12)$ \\
\hline & No & 2 & 86 & 1 & 1 & 1 & 1 & \\
\hline \multirow[t]{2}{*}{ Comorbidity } & Yes & 15 & 53 & 20.67 & 0.000 & 12.9 & 0.003 & $(2.45-67.60)$ \\
\hline & No & 2 & 146 & I & 1 & 1 & 1 & \\
\hline
\end{tabular}


intensive phases of TB treatment. Similar to the finding of this study, the onset of developing drug induced hepatotoxicity conducted in Dawro zone, Ethiopia was ranging from 13-58 days of starting the first-line treatment. ${ }^{11}$ However, according to the study done in Morocco, the onset of anti-TB drug induced hepatotoxicity was not in line with the present study which was reported as 10-25 days. ${ }^{15}$ In addition, the duration of developing hepatotoxicity in a study conducted in Egypt was not concordant with the present study. ${ }^{17}$ Generally, even though the days of developing hepatotoxicity among cases of different studies is different, the patients developed hepatotoxicity in the intensive phases of the TB treating protocol where adaptive mechanisms of the liver are not developed against TB drugs. In line with the study conducted in Egypt, ${ }^{17}$ in the present study, the liver function of the patients developing hepatotoxicity became normal after 2 weeks of cessation of therapy.

In the present study, the patients who developed hepatotoxicity to first-line TB therapy were subjected and decided to management of their hepatotoxicity as the ALT/AST level was raised to at least three times ULN and manifested the sign symptoms of hepatotoxicity. ${ }^{5}$ The patients developing hepatoxicity were managed following the advice of the International Union against $\mathrm{TB}$ and Lung Disease and WHO. ${ }^{16}$ Accordingly, patients developing hepatotoxicity were allowed to stop taking their treatment regimen for 2 weeks until their liver function became normal. Then, after normalization of the liver function, the TB of the cases was managed by giving the same dosage and types of the first-line drugs simultaneously without modification. In spite of this protocol, on follow-up, there was no recurrence of drug induced hepatotoxicity among all the cases managed. However, this management of TB among patients developing hepatotoxicity is against the American and British thoracic societies' guidelines and/or management principles where the anti-TB drugs is reinitiated consecutively starting from the safest drugs. ${ }^{5,18}$ Generally, the application of rechallenging anti-TB drugs for liver injured patients simultaneously or consecutively should consider the benefit risk condition of treating TB for the community, reducing the, emergence of multidrug resistant TB and the health condition of the individual patient.

In this study having extra pulmonary $\mathrm{TB}$, old age and having comorbidity were found to be significant predictors in the development of anti-TB drugs induced hepatotoxicity on multivariable logistic regression analyses.

The likelihood of developing hepatotoxicity to anti-TB drugs was 0.85-times lower in patients aged from 18-49 years old compared to those aged 65 or above. This finding is supported by studies done in southeast Iran, ${ }^{12}$ Canada, ${ }^{19}$ and Spain. ${ }^{14}$ No association was reported between age- and druginduced hepatotoxicity in many studies. ${ }^{11,14,20}$ Higher incidence of drug-induced hepatotoxicity in older age may be due to the presence of co-morbid diseases and use of additional drugs to treat these comorbidities in the older age group.

Patients with pulmonary TB had 0.26-times lower risk of developing hepatotoxicity to anti-TB drugs compared to those with extrapulmonary TB. Extrapulmonary organ involvement was reported to be associated with the incidence of anti-TB drug induced hepatotoxicity in studies done in a Nepalese population ${ }^{21}$ and India. ${ }^{22,23}$ The increased incidence of anti-TB drug induced hepatotoxicity in patients with extra pulmonary TB may be attributed to the fact that extrapulmonary TB indicates the severe form of the disease and the liver may be one of the organs affected.

Patients who had a comorbid medical condition were 12.9-times more likely to experience hepatotoxicity compared to TB patients who had no comorbidity. This finding is supported by different studies. ${ }^{24-26}$ This may be attributed to the use of concomitant drugs for comorbid condition and decreased metabolism and excretion of drugs in patients with comorbid medical conditions.

Alcohol consumption is a well-established risk factor for anti-TB drug-induced hepatotoxicity, as shown in studies done at Dawro zone, ${ }^{11}$ southeastIran, ${ }^{12}$ and a Nepalese population. ${ }^{21}$ In our study, however, the is no significant association between alcohol consumption and drug-induced hepatotoxicity in multivariate analysis. This might be due to the difference in the levels of alcohol consumption among study populations of the different studies in different countries.

\section{Limitation of the Study}

The present study was not able to assess the occurrence or the reoccurrence of hepatotoxicity in the continuous phases of treatment of TB because of the shortage of time and withdrawal of participants from the study.

\section{Conclusions}

The incidence of hepatotoxicity is relatively high among TB patients taking first-line anti-TB drugs. Thus, the liver function of patients with old age, comorbid diseases, and extrapulmonary TB should be regularly monitored to reduce the severity of drug-induced hepatotoxicity.

\section{Data Sharing Statement}

The data is available in the public library of Debre Markos University. 


\section{Ethics Approval and Consent to Participate}

The ethical clearance was obtained from the Institutional Research Ethics Review Committee of College of Health science, Debre Markos University. An official letter was written to the study setting including Debre Markos, Mota, and Bichena Hospitals to address the administrative issues. This study was conducted in accordance with the Declaration of Helsinki ethical principles, ${ }^{27}$ where verbal consent was taken from each participant. First the prepared verbal consent was reviewed and approved by the Institutional Research Ethics Review Committee of College of Health science, Debre Markos University. Then, the study participants were asked verbally whether they are willing to participate or not in the study by explaining its purpose and confidentiality. The general purpose of the research was explained with the local language, Amharic, to the participants before starting the data and blood sample collection. For confidentiality reasons, the participant's name was not included in the consent form; instead a code was used for identification purposes. Each participant was informed about his/her right of withdrawing from the study without restriction whenever necessary. The verbally obtained informed consent was documented as that of the individual data collection questionnaire because it was filled out as "Yes" or "No" alternatives by the interviewer.

\section{Author Contributions}

All authors made substantial contributions to the conception and design, acquisition of data, or analysis and interpretation of data; took part in drafting the article or revising it critically for important intellectual content; agreed to submit to the current journal; gave final approval of the version to be published; and agree to be accountable for all aspects of the work.

\section{Disclosure}

The authors declare that they have no competing interests.

\section{References}

1. CDC. National Notifiable Disease Surveillance System. Tuberculosis (TB): (Mycobacterium tuberculosis) case definition; 2009.

2. CDC. How TB spreads. 2016.

3. Riccardi N, Cenderello G, Borroni E, Rutigliani M, Cirillo DM. A case report of mucocutaneous tuberculosis after orthotopic liver transplantation: a challenging diagnosis. BMC Infect Dis. 2018;18(1):1-4.
4. Tostmann A, Boeree MJ, Aarnoutse RE, De Lange WC, Van Der Ven AJ, Dekhuijzen R. Antituberculosis drug-induced hepatotoxicity: concise upto-date review. J Gastroenterol Hepatol. 2008;23(2):192-202.

5. Saukkonen JJ, Cohn DL, Jasmer RM, et al. An official ATS statement: hepatotoxicity of antituberculosis therapy. Am J Respir Crit Care Med. 2006;174(8):935-952.

6. Marzuki O, Fauzi A, Ayoub S, Kamarul Imran M. Prevalence and risk factors of anti-tuberculosis drug-induced hepatitis in Malaysia. Singapore Med J. 2008;49(9):688.

7. Yee D, Valiquette C, Pelletier M, Parisien I, Rocher I, Menzies D. Incidence of serious side effects from first-line antituberculosis drugs among patients treated for active tuberculosis. Am J Respir Crit Care Med. 2003;167(11):1472-1477.

8. Riccardi N, Alagna R, Saderi L, et al; for StopTB Italia Onlus Group. Towards tailored regimens in the treatment of drug-resistant tuberculosis: a retrospective study in two Italian reference Centres. $B M C$ Infect Dis. 2019;19(1):564.

9. Abbasi MA, Ahmed N, Suleman A, et al. Common risk factors for the development of anti tuberculosis treatment induced hepatotoxicity. J Ayub Med Coll Abbottabad. 2014;26:3.

10. Yimer G, Aderaye G, Amogne W, et al. Anti-tuberculosis therapy-induced hepatotoxicity among Ethiopian HIV-positive and negative patients. PLoS One. 2008;3(3):e1809.

11. Abera W, Cheneke W, Abebe G. Incidence of antituberculosis-druginduced hepatotoxicity and associated risk factors among tuberculosis patients in Dawro Zone, South Ethiopia: a cohort study. Int J Mycobacteriol. 2016;5(1):14-20.

12. Metanat M, Mood BS, Salehi M, Rakhshani M, Metanat S. Risk factors and pattern of changes in liver enzymes among the patients with anti-tuberculosis drug-induced hepatitis. Int J Infect. 2015;2(2): e25753.

13. Ungo JR, Jones D, Ashkin D, et al. Antituberculosis drug-induced hepatotoxicity: the role of hepatitis $\mathrm{C}$ virus and the human immunodeficiency virus. Am J Respir Crit Care Med. 1998;157(6):1871-1876.

14. Fernandez-Villar A, Sopena B, Fernandez-Villar J, et al. The influence of risk factors on the severity of anti-tuberculosis drug-induced hepatotoxicity. Int J Tuberc Lung Dis. 2004;8(12):1499-1505.

15. El Bouazzi O, Hammi S, Bourkadi JE, et al. First line anti-tuberculosis induced hepatotoxicity: incidence and risk factors. Pan Afr Med J. 2016;25.

16. Ramappa V, Aithal GP. Hepatotoxicity related to anti-tuberculosis drugs: mechanisms and management. J Clin Exp Hepatol. 2013;3(1):37-49.

17. Makhlouf HA, Helmy A, Fawzy E, El-Attar M, Rashed HAG. A prospective study of antituberculous drug-induced hepatotoxicity in an area endemic for liver diseases. Hepatol Int. 2008;2(3):353.

18. Society JTCotBT. Chemotherapy and management of tuberculosis in the United Kingdom: recommendations 1998. Thorax. 1998;53 (7):536-548.

19. Devarbhavi H. Antituberculous druginduced liver injury: current perspective. Trop Gastroenterol. 2011;32(3):167-174.

20. Hassen Ali A, Belachew T, Yami A, Ayen WY. Anti-tuberculosis drug induced hepatotoxicity among TB/HIV co-infected patients at Jimma University Hospital, Ethiopia: nested case-control study. PLoS One. 2013;8(5):e64622.

21. Shaky R, Shrestha B. Evaluation of risk factors for antituberculosis drugs-induced hepatotoxicity in Nepalese population. Kathmandu Univ Med Sci Eng Technol. 2006;2(1).

22. Parthasarathy R, Sarma GR, Janardhanam B, et al. Hepatic toxicity in South Indian patients during treatment of tuberculosis with short-course regimens containing isoniazid, rifampicin and pyrazinamide. Tubercle. 1986;67:99-108.

23. Sharma SK, Balamurugan A, Saha PK, Pandey RM, Mehra NK. Evaluation of clinical and immunogenetic risk factors for the development of hepatotoxicity during antituberculosis treatment. Am J Respir Crit Care Med. 2002;166:916-919. 
24. Warmelink I, Nick H, van der Werf TS, van Altena R. Weight loss during tuberculosis treatment is an important risk factor for drug induced hepatotoxicity. Br J Nutr. 2011;105:400-408.

25. Yew WW, Chang KC, Chan DP. Oxidative stress and first line antituberculosis induced hepatotoxicity. Antimicrob Agents Chemother. 2018;62(10):e011633-18.
26. Li X, Gao P, Niu J. Metabolic comorbidities and risk of developement and severity of drug induced liver injury. Biomed Res Int. 2019;2019.

27. World Medical Association. World Medical Association declaration of helsinki. Ethical principles for medical research involving human subjects. Bull World Health Organ. 2001;79(4):373.

\section{Publish your work in this journal}

Hepatic Medicine: Evidence and Research is an international, peerreviewed, open access journal covering all aspects of adult and pediatric hepatology in the clinic and laboratory including the following topics: Pathology, pathophysiology of hepatic disease; Investigation and treatment of hepatic disease; Pharmacology of drugs used for the treatment of hepatic disease. Issues of patient safety and quality of care will also be considered. The manuscript management system is completely online and includes a very quick and fair peer-review system, which is all easy to use. Visit http://www.dovepress.com/ testimonials.php to read real quotes from published authors. 